\title{
An Extension of the Linkwitz-Riley Crossover Filters for Audio Systems and Their Sampled Data Implementation
}

\author{
fred harris, Elettra Venosa, Xiaofei Chen, Prafulla \\ Muthyala \\ Electrical and Computer Engineering Department \\ San Diego State University \\ San Diego, USA
}

\author{
Chris Dick \\ Xilinx Corp. \\ San Jose, USA
}

\begin{abstract}
Loud speaker drivers are not able to operate over the entire audio spectrum. The standard response to this limitation is to use multiple drivers designed to operate over selected contiguous frequency spans such as low-pass, band-pass and high-pass. Crossover filters partition the audio spectrum into appropriate spectral bands matched to the bandwidth of the multiple speaker drivers. For many years, the standard crossover filters that perform the spectral partition has been the LinkwitzRiley (L-R) Filter [1]. In this paper we review the structure of the L-R filters, extend their design to any order crossover, and present their efficient DSP implementations.
\end{abstract}

Index Terms - Linkwitz-Riley crossover filters; DSP crossover filters; two-path crossover filters.

\section{INTRODUCTION}

Loudspeakers are mechanical electro acoustic transducers that convert electrical signals to acoustic sound pressure levels that we perceive as sound. The range of frequencies to be reproduced by a loudspeaker system spans 4-decades, typically $20 \mathrm{~Hz}$ to $20,000 \mathrm{~Hz}$. The large stiff paper cone of a speaker driver designed to operate at long wavelength, low audio frequency, will buckle when attempting to operate at short wavelength, high audio frequency. The buckling supports standing waves on the cone which introduces acoustic distortion. To avoid this distortion multiple speaker drivers, of successively reduced dimensions, designed to operate in different frequency ranges are used to span audio spectrum. Crossover filters partition the spectrum of the input electrical signal into sub bands matched to the frequency response bands of the separate loudspeaker drivers. Figure 1 illustrates possible signal flow options for the amplifier(s), filters, and loudspeaker drivers for a low and high frequency crossover.

We might consider 2-nd order Butterworth filters for the low-pass and high-pass filters. These have spectral roll of rates of $40 \mathrm{~dB}$ per decade and should offer reasonable decoupling between the low-pass and high-pass spectra. Figure 2 presents the spectra of a pair of 2-nd order Butterworth filters. We note that the two filters cross at their $3-\mathrm{dB}$ attenuation frequency and that there is hope that the signals at the crossover add their contribution from the two filters. The signals separated by the electrical filters are combined acoustically in the air column beyond the face of the loudspeaker enclosure. As we see in the center sub figure of Fig. 2, the sum of the acoustic signals do not sum to a flat response at the filter crossover frequency but rather exhibits a spectral null. This is because the two signal components at the cross over frequency while equal in amplitude differ by 180 degree phase shift. This can be seen in the sum of the low-pass and high pass transfer functions as shown in (1).

$$
\begin{gathered}
H_{L P}(S)=\frac{\omega_{0}^{2}}{S^{2}+2 \zeta \omega_{0} S+\omega_{0}^{2}} \\
H_{H P}(S)=\frac{S^{2}}{S^{2}+2 \zeta \omega_{0} S+\omega_{0}^{2}} \\
H_{H P}(S)+H_{L P}(S)=\frac{S^{2}+\omega_{0}^{2}}{S^{2}+2 \zeta \omega_{0} S+\omega_{0}^{2}} \\
H_{H P}(S)-H_{L P}(S)=\frac{S^{2}-\omega_{0}^{2}}{S^{2}+2 \zeta \omega_{0} S+\omega_{0}^{2}}
\end{gathered}
$$
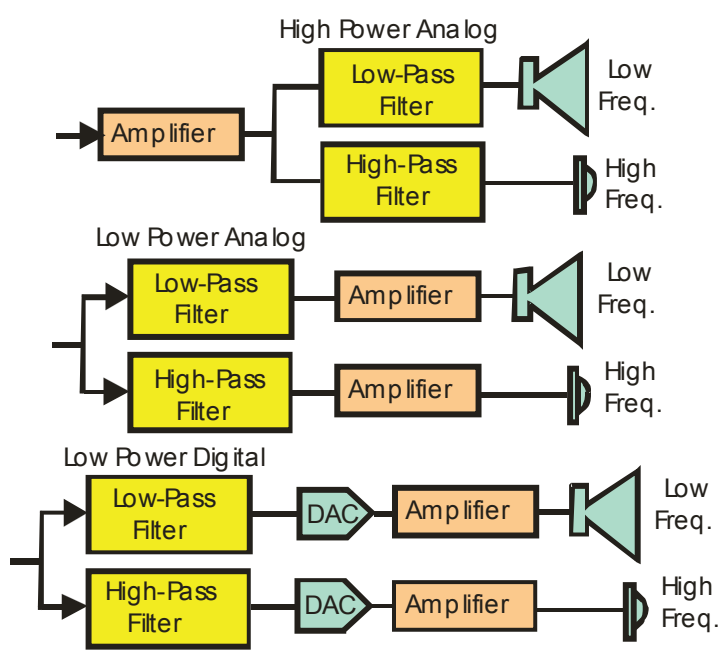

Figure 1. Two Path Filter Structures.

U.S. Government work not protected by U.S. copyright 

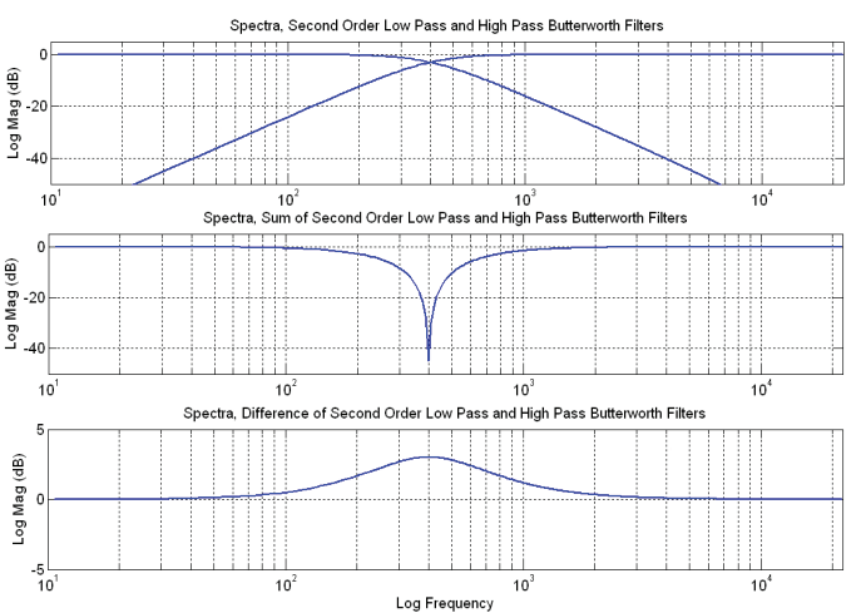

Figure 2. Spectra of 2-nd Order Low-Pass and High-Pass Filters and Their Sum and Difference of Their Time Responses.

Here we see numerator of the sum transfer function has zeros on the $\mathrm{j} \omega$ axis at the crossover frequency $\omega_{0}$. We can avoid the spectral notch due to the zeros of the frequency axis by reversing the polarity of either of the filtered signals. Under this condition, the difference numerator has real zeros and the composite gain at the $3 \mathrm{~dB}$ crossover frequency, as seen in Fig. 1, is seen to be $3 \mathrm{~dB}$.

\section{THE LINKWITZ-RILEY CROSSOVER FILTER}

The LR crossover filter system can be described from a number of perspectives, but the most straight forward perspective is that it uses a cascade of two low-pass and two high-pass Butterworth filters for the crossover filters. In the original work two 1-st order Butterworth filters formed 2-nd order crossover filters (with repeated roots) and two 2-nd order Butterworth filters (with repeated roots) formed 4-th order crossover filters. The first descriptive change we note when we use a cascade of Butterworth filters is that at their crossover frequency, the filters are down 6-dB rather than 3$\mathrm{dB}$. The second descriptive change is that, due to the higher order filter, the cascade of two 2-nd order filters falls at $80 \mathrm{~dB}$ per decade.

Equation 2 shows the transfer function of the LR crossover filters with repeated 2-nd order Butterworth low-pass and high-pass filters

$$
\begin{gathered}
H_{L R-L P}(S)=\left[\frac{\omega_{0}^{2}}{S^{2}+2 \zeta \omega_{0} S+\omega_{0}^{2}}\right]^{2} \\
H_{L R-H P}(S)=\left[\frac{S^{2}}{S^{2}+2 \zeta \omega_{0} S+\omega_{0}^{2}}\right]^{2} \\
H_{L R-H P}(S)+H_{L R-L P}(S)=\frac{S^{4}+\omega_{0}^{4}}{\left[S^{2}+2 \zeta \omega_{0} S+\omega_{0}^{2}\right]^{2}}
\end{gathered}
$$

We note that the denominator of (2) has the repeated roots of the 2-nd order Butterworth filter, the poles on the $\omega_{0}$ circle at \pm 135 degrees from the real axis. These poles are the LHP roots of the 4-th order denominator polynomial of (3), when $\mathrm{N}=2$. The numerator of (2) is precisely the denominator of (3) with $\mathrm{N}=2$. The numerator of (2) contains all 4-roots of denominator (2), the zeros on the $\omega_{0}$ circle at \pm 45 and \pm 135 degrees from the real axis. This factoring is shown in (4) and is illustrated in Fig. 3. We note that the LHP zeros of (2) cancel one of the pair of repeated poles in (2) leaving mirror image pole-zero pairs about the imaginary axis. The sum transfer function is seen to be an all-pass filter with constant unity gain at all frequencies.

We note that this all-pass relationship is valid for a repeated Butterworth filter of any order, such as 1, 2, 3, 4, 5, 6, etc. When the order of the filter is odd, such as 1,3 , or 5 , we invert the signal polarity on one of the two crossover filters to align the zeros with the repeated poles on the real axis and thus form a sum filter with the desired all-pass characteristics.

$$
\begin{gathered}
\mid H_{N}\left(\left.S\right|^{2}=\frac{1}{1+\left(S / \omega_{0}\right)^{2 N}}=\frac{\left(\omega_{0}\right)^{2 N}}{S^{2 N}+\left(\omega_{0}\right)^{2 N}}\right. \\
H_{H P}(S)+H_{L P}(S)=\frac{S^{4}+\omega_{0}^{4}}{\left[S^{2}+2 \zeta \omega_{0} S+\omega_{0}^{2}\right]^{2}} \\
=\frac{S^{2}+2 \zeta \omega_{0} S+\omega_{0}^{2}}{\left[S^{2}+2 \zeta \omega_{0} S+\omega_{0}^{2}\right]\left[S^{2}+2 \zeta \omega_{0} S+\omega_{0}^{2}\right.} \frac{\left.S_{0} S+\omega_{0}^{2}\right]}{\left[S^{2}\right]}
\end{gathered}
$$

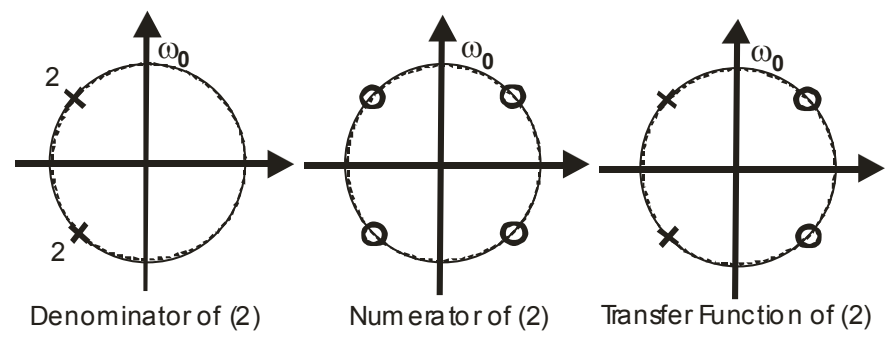

Figure 3. Poles of Equation 2, Zeros of Equation 2, and Poles and Zeros of Equation 2s.

\section{SAMPLE DATA L-R CROSSOVER FILTERS}

The repeated Butterworth filters required to implement the crossover filters in the LR system can be converted from the continuous domain to the sampled data domain by the standard Bilinear transform. We start with the Butterworth poles positioned in the LHP on the unit bandwidth circle in the complex S-plane and separated by angles $2 \pi /(2 \mathrm{~N})$. We predistort the analog bandwidth by scaling the circle to $\operatorname{Tan}\left(\pi \mathrm{f}_{0} / \mathrm{f}_{\mathrm{S}}\right)$ and then move the scaled roots to their image in the complex Z-plane with the mapping shown in (5). Note that the $D C$ zeros $S=0$ are mapped to $Z=-1$, and the zeros $S=\infty$ are mapped to $Z=+1$. The $Z$-plane transfer functions must be scaled for unity gain at $Z=+1$ for the low-pass filter and at $Z$ $=-1$ for the high pass filter. This standard transformation is 
embedded in many filter design packages such as the one in MATLAB.

$$
S=S_{k} \Rightarrow Z_{k}=\frac{1+S_{k}}{1-S_{k}}
$$

Since the Butterworth design routine is readily available, we can simply call the design routine to extract the coefficients of the Butterworth transfer function. We examine a 4-th order Butterworth filter for our first example. The 2-nd order numerator and denominators for the two 2-nd order sections in each of the 4-th order Butterworth low-pass and high-pass filters is shown in (6).

$[\mathrm{b} 1, \mathrm{a} 1]=\operatorname{butter}(4,2000 / 22050)$;

$\mathrm{SOS}=\operatorname{tr} 2 \operatorname{sos}(\mathrm{b} 1, \mathrm{a} 1)$;

$$
\begin{aligned}
& \text { b11 }=1.000000 \quad 2.0000000 \quad 1.000000 ; \\
& \mathrm{g} 11=0.016005 \\
& \mathrm{a} 11=1.000000-1.5236413 \quad 0.587663 ; \\
& \mathrm{b} 12=1.000000 \quad 2.0000000 \quad 1.000000 ; \\
& \mathrm{g} 12=0.018204 \\
& \mathrm{a} 12=1.000000 \quad-1.7329280 \quad 0.805744
\end{aligned}
$$

[b2,a2] = butter(2,2000/22050,'high');

$$
\begin{aligned}
& \mathrm{SOS}=\operatorname{tr} 2 \operatorname{sos}(\mathrm{b} 2, \mathrm{a} 2) \text {; } \\
& \text { b21 }=1.000000-2.0000000 \quad 1.000000 \text {; } \\
& \mathrm{g} 21=0.777826 \\
& \mathrm{a} 12=1.000000-1.5236413 \quad 0.587663 \text {; } \\
& \text { b22 }=1.000000-2.0000000 \quad 1.000000 \text {; } \\
& \mathrm{g} 22=0.884668 \\
& \mathrm{a} 22=1.000000 \quad-1.7329280 \quad 0.805744
\end{aligned}
$$
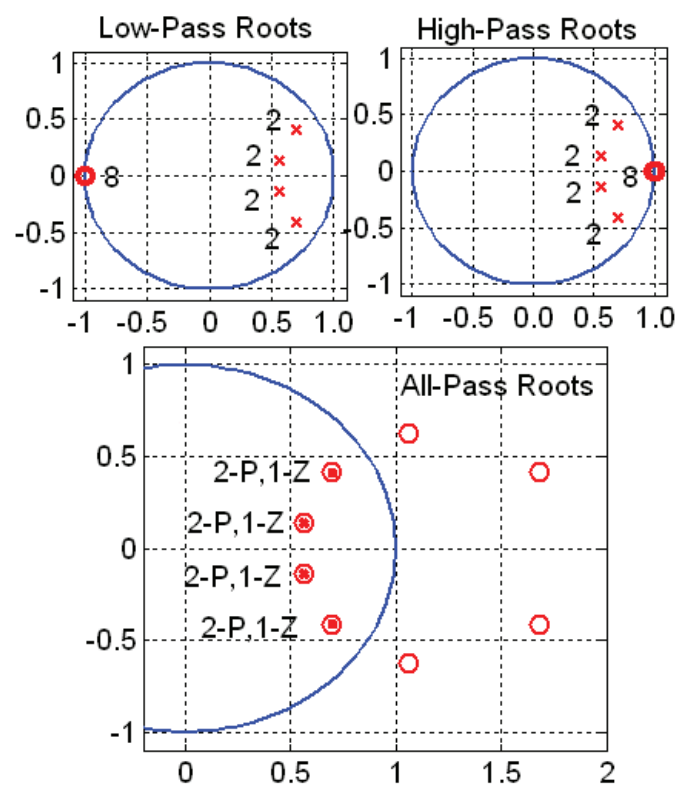

Figure 4. Pole Zero Diagrams for Sampled Data Filters of 4-th Order and 8-th Order 2-kHz L-R Crossover Filters
As expected, the denominators are the same for the low-pass and high-pass filters and the numerators are the same except for the sign of the b1 coefficient [llllll $\left.\begin{array}{lll}1 & 1\end{array}\right]$ and $\left[\begin{array}{lll}1 & -2 & 1\end{array}\right]$. The primary difference in the coefficient list is the scaling terms $\mathrm{g}_{\mathrm{ik}}$. Figure 4 shows the pole-zero diagrams for the L-R Butterworth low-pass and high-pass filters and for the summed all-pass filter.

Figure 5 shows the spectra of the crossover filters and their sum for the L-R crossover filter of order eight and of order four which is shown for comparison.
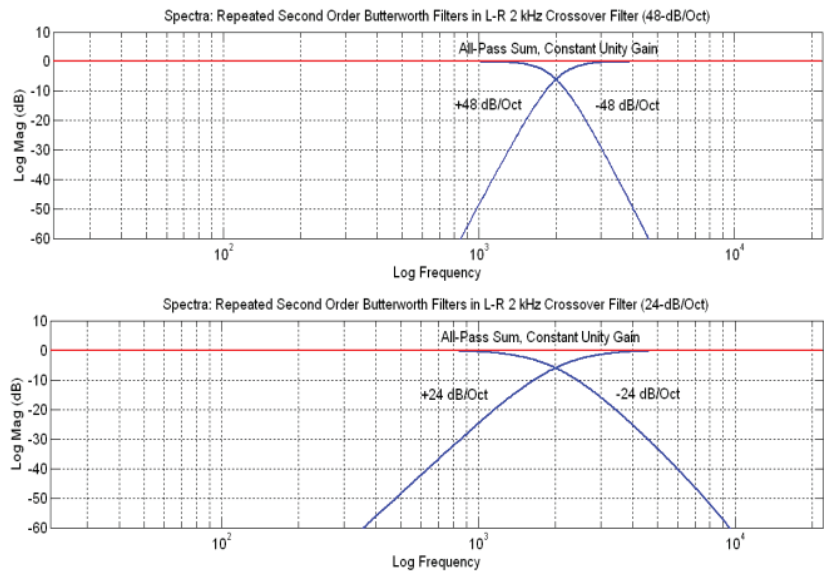

Figure 5. Spectra of Fourth Order and Eighth Order 2-kHz L-R Crossover Filters

\section{FIRST L-R FILTER IMPLEMENTATION OPTION}

While the 1-st and 2-nd order canonic filters, usually identified as the bi-quadratic filter, is a common low-order building block used to implement higher recursive filters it is not the only option. An alternate, and very effective, low order building block set are the 1-st-order and 2-nd order all-pass filter [2] sets shown in Fig. 8. Note that in this architecture, each coefficient is in the feedback path and in the feed forward path so that a single coefficient forms both a pole and its reciprocal zero.

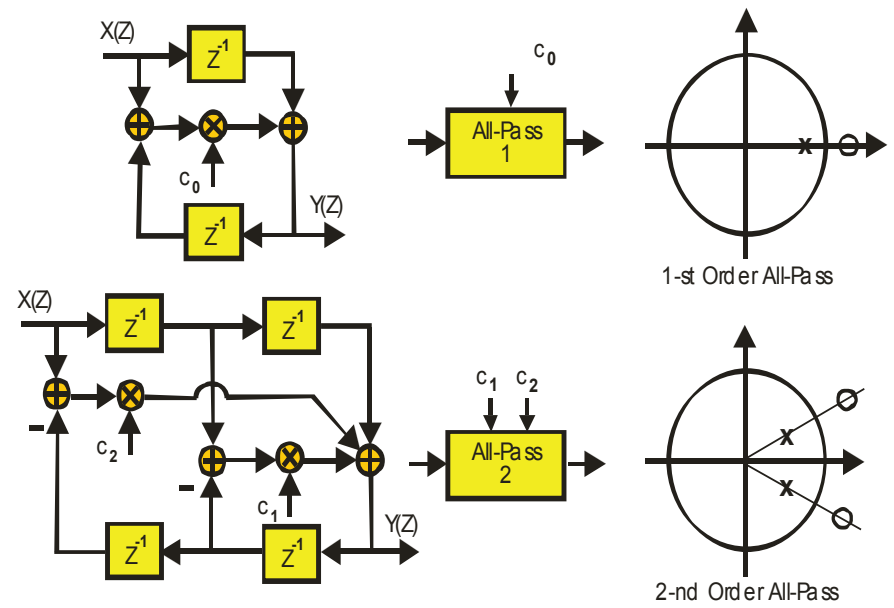

Figure 8. Block Diagram: 1-st Order and 2-nd Order All-Pass Filters 
Since these filters are all-pass, with unity gain, they do not require scaling factors. Figure 9 forms the low-pass and highpass versions of the 5-th order Butterworth filter as the sum and difference of the two path arms containing the all-pass filters of Fig. 8. Notice that the scaling factor required to cancel the gain of at the output sum is trivially $1 / 2$ and does not contribute to the arithmetic workload. This filter offers 1-pole and 1-zero per coefficient and does not require scaling coefficients.
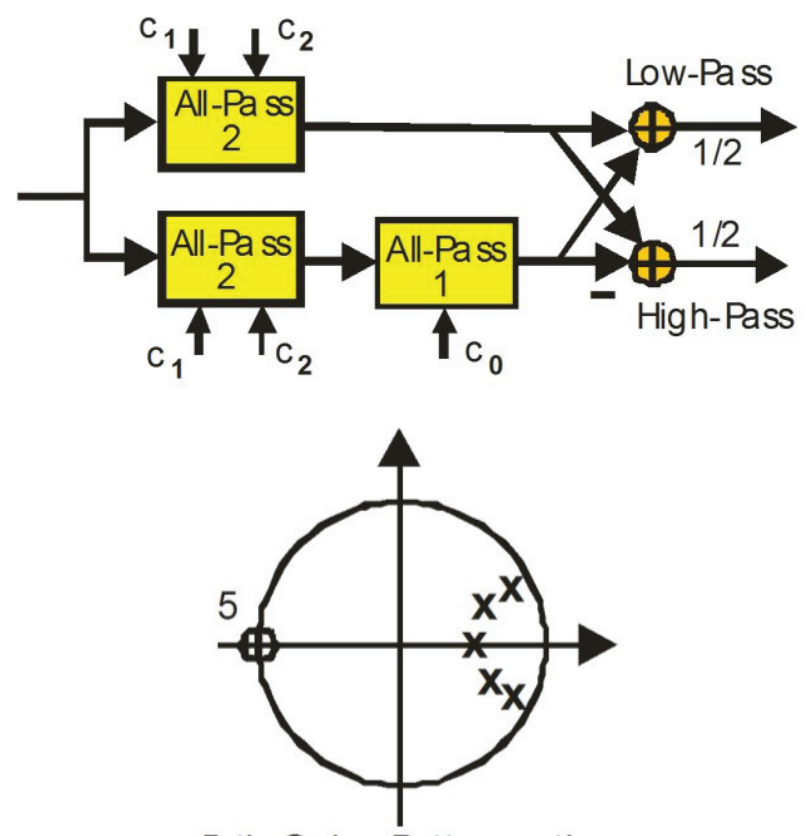

\section{5-th Ord er Butterworth}

Figure 9. 2-Path All-Pass Sum and Difference form 5-th Order Low-Pass and High Pass Butterworth Filter

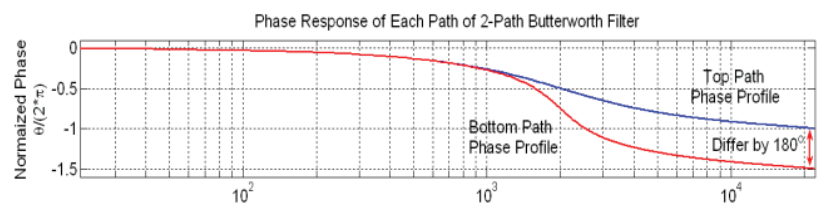

Phase Difference Between Two Paths of 2-Path Butterworth Filter
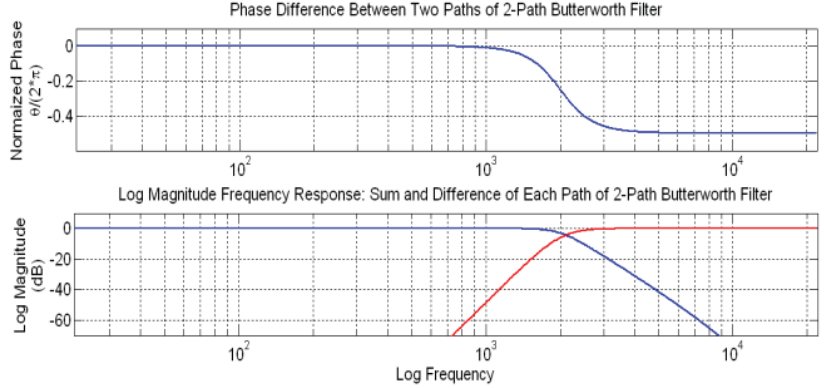

Figure 10. Phase Profiles and Phase Difference of 2-Path Filter, and Frequency Response of Sum and Difference of 2-Path Signals
Figure 10 presents phase profiles of the two path filter of Fig. 9. Here we see phases in the two paths are aligned in the passband frequency span and they differ by approximately 180 degree in the stop-band frequency span, achieving exactly 180 phase difference at the half sample rate. Finally Fig. 11 presents the block diagram of a 10-th order L-R crossover filter using only three of the two-path 5-th order Butterworth Filters. The sum and difference of the 2-path input filter present both low-pass and high-pass responses. Note that filter only requires 15 coefficients to build a 10 -th order L-R filter as opposed to the bi-quadradic implementation that required 24 coefficients to build an 8-th order L-R filter.

10-th Order Linkwitz-Rile y Crossover Filter

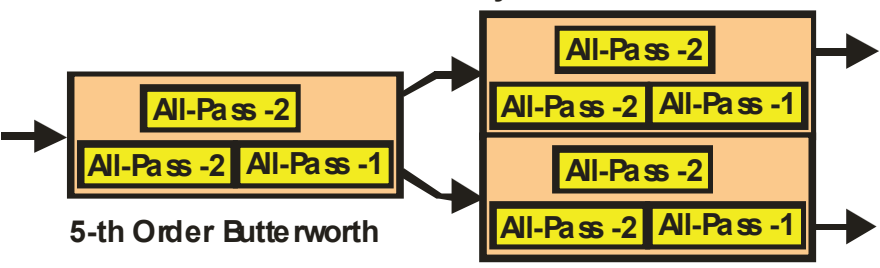

Figure 11. Two-Path All-Pass Butterworth Filters and 10-th Order L-R Crossover Filter Containing three 5-th Order Butterworth Filters.

\section{Closing COMMENTS}

We have shown that L-R filters of any even order can be formed by Butterworth filters of any order. We also showed that odd order Butterworth filters implemented as 2-path allpass filters required significantly less multiplies than canonic bi-quadratic filters.

\section{REFERENCES}

[1] Sigfied Linkwitz and Russ Riley, Passive Crossover Networks for Noncoincident Drivers in JAES, Volume 26, Number 3, pp 149-150, March 1978.

[2] fred j. harris, "Multirate Signal Processing for Communication Systems", Prentice Hall, 2004.

[3] P.P. Vaidyanathan, "Multirate Systems and Filter Banks", Pren tice-Hall, Englewood Cliffs, 1993. 